\title{
Study of the Dynamic Characteristics of Ball Screw with a Load Disturbance
}

\author{
Zhe Du, ${ }^{1}$ Xiao-Lan Zhang, ${ }^{1}$ and Tao Tao ${ }^{2,3}$ \\ ${ }^{1}$ Henan University of Science and Technology, Luoyang 471023, China \\ ${ }^{2}$ Xian Jiaotong University, Xian 710049, China \\ ${ }^{3}$ State Key Laboratory for Manufacturing Systems Engineering, Xian Jiaotong University, Xian 710049, China \\ Correspondence should be addressed to Tao Tao; taotao@mail.xjtu.edu.cn
}

Received 21 December 2015; Revised 6 May 2016; Accepted 22 May 2016

Academic Editor: Alessandro Gasparetto

Copyright (C) 2016 Zhe Du et al. This is an open access article distributed under the Creative Commons Attribution License, which permits unrestricted use, distribution, and reproduction in any medium, provided the original work is properly cited.

\begin{abstract}
The dynamic character of ball screw is the key factor that influences the machining accuracy of numerical control (NC) machine tool. To improve the dynamic characteristics of the NC machine tool, it is necessary to study the dynamic characteristics of a ball screw. In this paper, the kinematics of a ball screw mechanism (BSM) are studied to expound the dynamic process of the drive, and the load disturbance is considered to analyze the contact deformation based on the Hertzian contact theory. The velocity relationships among the ball, screw, and nut are analyzed, and the influence of the contact deformation on the dynamic characteristics is simulated and investigated experimentally. The results show that the relationships between the contact deformation, which is affected by the material characteristics, the contact angle, and the load of nut are nonlinear. The contact deformation is a factor that cannot be ignored when considering the dynamic machining error of high-speed and high-precision machine tools.
\end{abstract}

\section{Introduction}

The numerical control (NC) machine tool is an important factor in the modern manufacturing industry, and the level of industrial development of a nation is much more dependent on the technology of NC machine tools. A ball screw is a precision transmission part of the feed unit of a machine tool, and its characteristics directly impact the precision of the machine tool. Although the linear motor is a new method to operate the feed unit, as a proven technique, the ball screw will play an important part in feed unit operations for a long time [1]. Currently, high-speed and high-precision machining techniques are thought to improve machining efficiency and precision; hence, it is necessary to intensely study the dynamic characteristics of ball screws.

The dynamic model, the modal parameter identification method, and the control character have been widely studied in previous works. Vicente et al. [2] present a high-frequency finite-dimension model of a ball screw in which the ball screw is considered as a continuous subsystem. In this model, the first model of a ball screw is rigid, and the axial and angular deformations only affect the mode in the second and fourth mode shapes, respectively. Varanasi and Nayfeh [3] present a distributed inertia model to study the dynamic characteristics of a ball screw in a closed loop control system. The finite righthalf plane zero in the transfer function between motor torque and carriage position is accurately predicted, and the significance of incorporating a viscoelastic damper into one of the lead-screw support bearings in the system transfer functions is noted. In a study by Okwudire [4], the deformation of a screw in a nut is considered with Timoshenko beam-shape functions for modeling the ball screw. Using this method, the identification precision of the natural frequency of the ball screw is improved. Okwudire and Altintas [5] study the lateral dynamic parameter, which is omitted in previous research, and show that the lateral dynamic parameter has an important influence on the dynamic characteristics of a ball screw. In these studies, the holistic dynamics of ball screws are studied meticulously; however, the characteristics of a combined system, including a ball, screw, and nut, are almost ignored.

Lin et al. [6] studied the ball screw mechanism theoretically with the aim of finding a mathematical description of the ball, screw, and nut kinematic and contact patterns. In that study, the slide phenomenon of a ball with a screw and 
nut is interpreted. In another paper, Lin et al. [7] analyze the factors that influence the efficiency of a ball screw and provide the formula for efficiency based on contact angle and helical angle. Wei and Lin [8] further study the BSM based on the research result of [6]. The influential parameters, such as the friction coefficient, normal force acting on the ball, and contact angle on a ball screw's mechanism at two contact areas, are simulated. Feng and Pan [9] use the vibrational signal of a nut (on a double nut and preload-adjustable ball screw feed drive system) to research the relationship between the preload and the vibration of a nut. Huang and Ravani [10] introduce the medial axis transform (MAT) method into the BSM analysis process. The force analysis of a ball, based on the kinematic and Hertzian contact, is provided. Cuttino et al. [11] research the nonlinear relationship between the load and deformation of a single nut ball screw contact. The instant stress state is analyzed, and the relationship of load and deformation is drawn. Mei et al. [12], taking the machining error of a ball screw into consideration, study the force distribution model of a ball based on the Hertzian contact theory.

The research presented above encompasses most of the dynamic and kinematic theory. However, the influence of the contact deformation on the motion precision of a ball screw is rarely investigated. In this study, the dynamic error of a ball screw is researched under a load disturbance condition that was partly based on the theoretical analyses developed by Lin et al. [6]. In Section 2, the kinematics of a ball screw are analyzed, and the velocity deviation between the ball and the screw is given in the absolute coordinate system. The force analysis of a ball is given in Section 3. The factors that influence the contact deformation are simulated in Section 4. The load disturbance experiments are implemented in Section 5. Finally, the conclusions are summarized in Section 6.

\section{Theoretical Analysis of BSM}

2.1. Motion Analysis of Ball Screw. The ball screw, which is composed of balls, a screw, and a nut, can achieve the transmission of linear motion and rotation. The analysis process is limited to the transmission from rotational motion to linear motion because that is the function of a ball screw in a machine tool. In the transmission process, the rotation of the screw compels the balls to move around the screw. At the same time, the balls generate a spinning motion. The drive torque from the motor causes not only the axial and angular deformation of the screw but also the contact deformation of the balls, the screw, and the nut, according to the Hertzian contact theory. Hence, the motion precision of the table will be influenced. To study the motion error caused by the contact deformation, the relative motion of the ball, screw, and nut should be analyzed first.

Three coordinate systems should be established, as shown in Figure 1. The first coordinate system, $X^{\prime}\left(x^{\prime}, y^{\prime}, z^{\prime}\right)$, is fixed with its $z^{\prime}$ axis accordant with the axis of the screw, and $x^{\prime} y^{\prime}$ plane is perpendicular to the screw axis. The second coordinate system, $X(x, y, z)$, is used to describe the rotation of the screw rotate $\Omega$ around $z^{\prime}$ axis. According to the

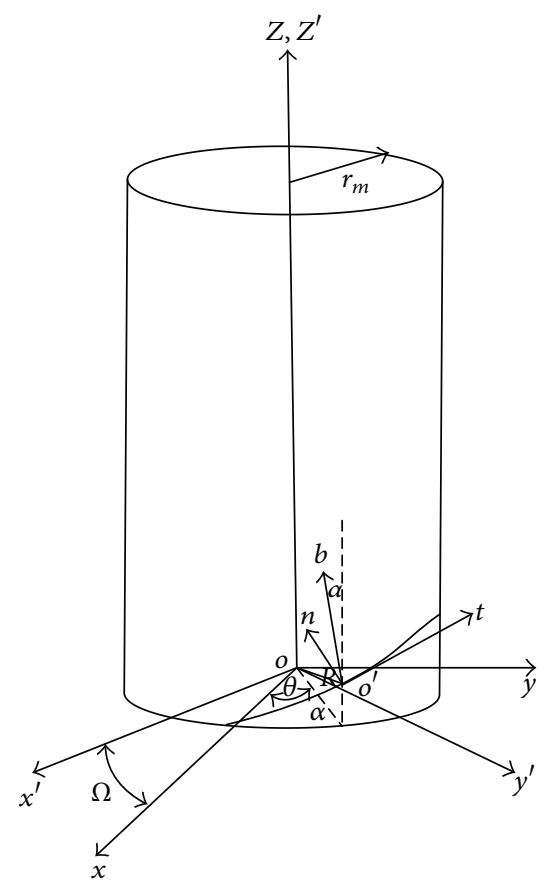

FIGURE 1: The position of the ball center in the chosen coordinate system.

rigid-body kinematics, the coordinate transformation relationship from $X$ to $X^{\prime}$ is

$$
\left[\begin{array}{l}
x^{\prime} \\
y^{\prime} \\
z^{\prime}
\end{array}\right]=T_{1}\left[\begin{array}{l}
x \\
y \\
z
\end{array}\right],
$$

where $T_{1}=\left[\begin{array}{ccc}C \Omega & -S \Omega & 0 \\ S \Omega & C \Omega & 0 \\ 0 & 0 & 1\end{array}\right]$ and $C \Omega=\cos \Omega, S \Omega=\sin \Omega$, as follows.

Assume that one of the balls moves an angle $\theta$ along the helical groove of the screw. So, the coordinate of the ball center in $X$ system is

$$
{ }^{r} P=\left[\begin{array}{lll}
r_{m} C \theta & r_{m} S \theta & r_{m} \theta t \alpha
\end{array}\right]^{T},
$$

where $t \alpha=L / 2 \pi r_{m}, r_{m}$ is the nominal radius of screw, $L$ is the pitch of screw, and $\alpha$ is helical angle of the groove.

In $X^{\prime}$ system the coordinate is

$$
\begin{aligned}
{ }^{w} P & =T_{1}\left[\begin{array}{lll}
r_{m} C \theta & r_{m} S \theta & r_{m} \theta t \alpha
\end{array}\right]^{T} \\
& =\left[\begin{array}{c}
r_{m} C \theta C \Omega-r_{m} S \theta S \Omega \\
S \Omega r_{m} C \theta+C \Omega r_{m} S \theta \\
r_{m} \theta t \alpha
\end{array}\right] .
\end{aligned}
$$

The Frenet coordinate system, $Y(t, n, b)$, is defined to describe the motion of the ball center along the groove. The origin is set on the ball center, $t$ axis is in the tangential direction of groove, and $n b$ plane is the cross section of the groove. 


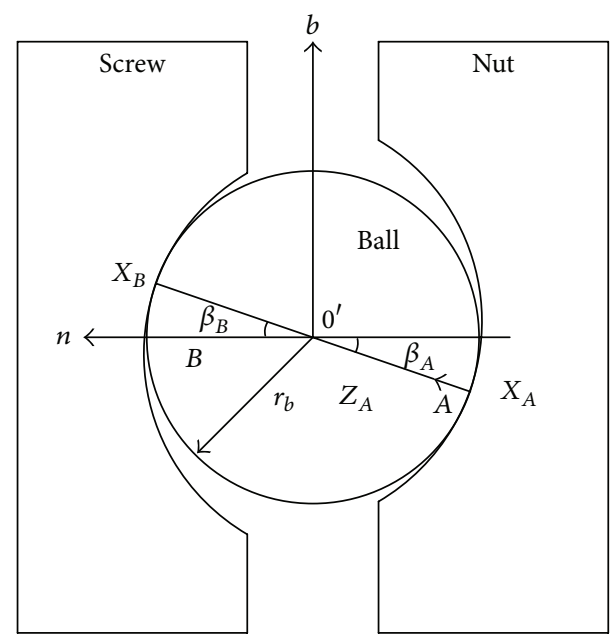

FIGURE 2: The contact point of the ball and the nut (screw), $A, B$, in the normal plane of the Frenet coordinates.

The Eulerian angle between $X$ and $Y$ is $[\pi / 2 \alpha \theta]$. The coordinate transformation relationship is

$$
\left[\begin{array}{l}
x \\
y \\
z
\end{array}\right]=P+\left[\begin{array}{ccc}
-C \alpha S \theta & -C \alpha C \theta & S \alpha \\
C \theta & -S \theta & 0 \\
S \alpha S \theta & S \alpha C \theta & C \alpha
\end{array}\right]\left[\begin{array}{l}
t \\
n \\
b
\end{array}\right] .
$$

The ball can only move relatively along $t$ direction of $Y$ system due to the restriction of the groove. Hence, the centers of the contact region are inevitably in $n b$ plane (Figure 2). The contact point between the ball and nut in $Y$ system is

$$
\left[\begin{array}{l}
t \\
n \\
b
\end{array}\right]_{A}=\left[\begin{array}{lll}
0 & r_{b} C \beta_{A} & r_{b} S \beta_{A}
\end{array}\right]^{T},
$$

and the contact point of the contact point between the ball and nut in $Y$ system is

$$
\left[\begin{array}{l}
t \\
n \\
b
\end{array}\right]_{B}=\left[\begin{array}{lll}
0 & -r_{b} C \beta_{B} & -r_{b} S \beta_{B}
\end{array}\right]^{T},
$$

where $\beta_{i}(i=A, B)$ is the contact angle between $n$ axis and the contact vector. $r_{b}$ is the radius of the ball.

Assume that the contact deformation has no influence on the ball center in $Y$ system, and the deformation can only affect the rotary angle of the screw and the nut position in $X^{\prime}$ system. Taking the contact deformation, $\delta_{j}(j=A, B)$, into consideration, the contact point position in $Y$ system is

$$
\begin{aligned}
& {\left[\begin{array}{l}
\bar{t} \\
\bar{n} \\
\bar{b}
\end{array}\right]_{A}=\left[\begin{array}{ll}
0 & \left(r_{b}-\delta_{A}\right) C \beta_{A}\left(r_{b}-\delta_{A}\right) S \beta_{A}
\end{array}\right]^{T},} \\
& {\left[\begin{array}{l}
\bar{t} \\
\bar{n} \\
\bar{b}
\end{array}\right]_{B}=\left[\begin{array}{ll}
0 & -\left(r_{b}-\delta_{B}\right) C \beta_{B}-\left(r_{b}-\delta_{B}\right) S \beta_{B}
\end{array}\right]^{T},}
\end{aligned}
$$

and the contact point position in $X^{\prime}$ system is

$$
\begin{gathered}
{\left[\begin{array}{l}
x \\
y \\
z
\end{array}\right]_{A}=T_{1}\left(P+T_{2} Y_{A}^{\prime}\right)=\left[\begin{array}{c}
r_{m}(C \theta C \Omega-S \theta S \Omega)+\left(r_{b}-\delta_{A}\right)\left(C \beta_{A} S \theta S \Omega-S \beta_{A} S \alpha C \Omega-C \beta_{A} C \alpha C \theta C \Omega\right) \\
r_{m}(C \theta S \Omega+S \theta C \Omega)+\left(r_{b}-\delta_{A}\right)\left(C \beta_{A} C \Omega S \theta-C \beta_{A} C \alpha C \theta S \Omega-S \beta_{A} S \alpha S \Omega\right) \\
r_{m} \theta t \alpha+\left(r_{b}-\delta_{A}\right)\left(C \beta_{A} S \alpha C \theta-S \beta_{A} C \alpha\right)
\end{array}\right],} \\
{\left[\begin{array}{l}
x \\
y \\
z
\end{array}\right]_{B}=T_{1}\left(P+T_{2} Y_{A}^{\prime}\right)=\left[\begin{array}{c}
r_{m}(C \theta C \Omega+S \theta S \Omega)+\left(r_{b}-\delta_{b}\right)\left(C \beta_{B} C \alpha C \theta C \Omega+S \beta_{B} S \alpha C \Omega+C \beta_{B} S \theta S \Omega\right) \\
r_{m}(C \theta S \Omega-S \theta C \Omega)+\left(r_{b}-\delta_{b}\right)\left(C \beta_{B} C \alpha C \theta S \Omega+S \beta_{B} S \alpha S \Omega+C \beta_{B} S \theta C \Omega\right) \\
r_{m} \theta t \alpha+\left(r_{b}-\delta_{b}\right)\left(S \beta_{B} C \alpha-C \beta_{B} S \alpha C \theta\right)
\end{array}\right] .}
\end{gathered}
$$

By the position analysis of the ball, screw, and nut in $X^{\prime}$ system, considering the contact deformation, the mutual affect will be analyzed in the following section.

2.2. Velocity Analysis of the Ball, Screw, and Nut. Mutual motion of the ball, screw, and nut, compelled by the drive torque, impacts the stability and precision of the transmission. It is necessary to study the motion process. First, assuming that the spinning angular velocity of the ball is $\vec{\omega}=\left[\begin{array}{lll}\omega_{t} & \omega_{n} & \omega_{b}\end{array}\right]^{T}, \omega_{i}(i=t, n, b)$ is the ball angular velocity around the $t, n, b$ axes of $Y$ system. The velocity of the ball at $B_{b}$ contact point in the $X$ system is

$$
\begin{aligned}
\dot{X}_{B}= & \dot{P}+\vec{\omega} \times Y_{B}^{\prime} \\
= & {\left[\begin{array}{c}
-r_{m} S \theta \\
r_{m} C \theta \\
r_{m} t \alpha
\end{array}\right] \dot{\theta} } \\
& +\left[\begin{array}{c}
-\left(r_{b}-\delta_{B}\right) S \beta_{B} \omega_{n}+\left(r_{b}-\delta_{B}\right) C \beta_{B} \omega_{b} \\
\left(r_{b}-\delta_{B}\right) S \beta_{B} \omega_{t} \\
\end{array}\right.
\end{aligned}
$$


In $X^{\prime}$ system, the velocity of the ball at $B_{b}$ contact point is

$$
\begin{aligned}
V_{B_{b}}= & T_{1} \dot{X}_{B}=\dot{T}_{1} X_{B}+T_{1} \dot{X}_{B} \\
= & \dot{\Omega}\left[\begin{array}{c}
-r_{m}(C \theta S \Omega+S \theta C \Omega)-\left(r_{b}-\delta_{B}\right)\left(C \beta_{B} C \alpha C \theta S \Omega-S \beta_{B} S \alpha S \Omega+C \beta_{B} S \theta C \Omega\right) \\
r_{m}(C \theta C \Omega+S \theta S \Omega)+\left(r_{b}-\delta_{B}\right)\left(C \beta_{B} C \alpha C \theta C \Omega-S \beta_{B} S \alpha C \Omega+C \beta_{B} S \theta S \Omega\right) \\
0
\end{array}\right]+\left[\begin{array}{c}
-r_{m}(S \theta C \Omega+C \theta S \Omega) \\
r_{m}(C \theta C \Omega-S \theta S \Omega) \\
r_{m} t \alpha
\end{array}\right] \dot{\theta} \\
+ & {\left[\begin{array}{c}
\left(r_{b}-\delta_{B}\right)\left(C \beta_{B} \omega_{b} C \Omega-S \beta_{B} \omega_{n} C \Omega-S \beta_{B} \omega_{t} S \Omega\right) \\
\left(r_{b}-\delta_{B}\right)\left(C \beta_{B} \omega_{b} S \Omega-S \beta_{B} \omega_{n} S \Omega+S \beta_{B} \omega_{t} C \Omega\right) \\
-\left(r_{b}-\delta_{B}\right) C \beta_{B} \omega_{t}
\end{array}\right] . }
\end{aligned}
$$

In $X^{\prime}$ system, the angular velocity of the screw is marked as $\left[\begin{array}{lll}0 & 0 & \dot{\Omega}\end{array}\right]^{T}$, and the velocity of the contact point on the screw, $B_{s}$, is

$$
\begin{aligned}
& =\left[\begin{array}{c}
0 \\
0 \\
\dot{\Omega}
\end{array}\right]\left[\begin{array}{c}
r_{m} C \theta+\left(r_{b}-\delta_{B}\right)\left(C \beta_{B} C \alpha C(\theta)-S \beta_{B} S \alpha\right) \\
r_{m} S \theta+\left(r_{b}-\delta_{B}\right) C \beta_{B} S(\theta) \\
r_{m} \theta t \alpha-\left(r_{b}-\delta_{B}\right)\left(C \beta_{B} S \alpha C(\theta)+S \beta_{B} C \alpha\right)
\end{array}\right] \\
& =\left[\begin{array}{c}
-r_{m} C \theta-\left(r_{b}-\delta_{b}\right)\left(C \beta_{B} C \alpha C \theta+S \beta_{B} S \alpha\right) \\
r_{m} S \theta+\left(r_{b}-\delta_{b}\right) C \beta_{B} S \theta \\
0
\end{array}\right] \dot{\Omega} .
\end{aligned}
$$

$$
V_{B_{s}}=\left[\begin{array}{c}
0 \\
0 \\
\dot{\Omega}
\end{array}\right] \times X_{B}
$$

Apparently, there are deviations between the ball velocity and the screw at the contact point $B$ :

$$
\begin{aligned}
\Delta V_{B b_{s}}= & {\left[\begin{array}{c}
-r_{m}(C \theta S \Omega+S \theta C \Omega+C \theta)-\left(r_{b}-\delta_{B}\right)\left(C \beta_{B} C \alpha C \theta(S \Omega-1)+S \beta_{B} S \alpha(S \Omega-1)+C \beta_{B} S \theta C \Omega\right) \\
r_{m}(C \theta C \Omega+S \theta S \Omega-S \theta)+\left(r_{b}-\delta_{B}\right)\left(C \beta_{B} C \alpha C \theta C \Omega-S \beta_{B} S \alpha C \Omega+C \beta_{B} S \theta(S \Omega-1)\right) \\
0
\end{array}\right] \dot{\Omega} } \\
& +\left[\begin{array}{c}
-r_{m}(S \theta C \Omega+C \theta S \Omega) \\
r_{m}(C \theta C \Omega-S \theta S \Omega) \\
r_{m} t \alpha
\end{array}\right] \dot{\theta}+\left[\begin{array}{c}
\left(r_{b}-\delta_{B}\right)\left(C \beta_{B} \omega_{b}-S \beta_{B} \omega_{n}\right) C \Omega-\left(r_{b}-\delta_{B}\right) S \beta_{B} \omega_{t} S \Omega \\
\left(r_{b}-\delta_{B}\right)\left(C \beta_{B} \omega_{b}-S \beta_{B} \omega_{n}\right) S \Omega+\left(r_{b}-\delta_{B}\right) S \beta_{B} \omega_{t} C \Omega \\
-\left(r_{b}-\delta_{B}\right) C \beta_{B} \omega_{t}
\end{array}\right] .
\end{aligned}
$$

The simulation result of [7] shows that the spinning velocity of the ball around $t$ axis, $\omega_{t}$, is so small that it can be omitted. Therefore, the approximated expression of (12) is

$$
\begin{aligned}
\Delta V_{B b_{s}}= & {\left[\begin{array}{c}
-r_{m}(C \theta S \Omega+S \theta C \Omega+C \theta)-\left(r_{b}-\delta_{B}\right)\left(C \beta_{B} C \alpha C \theta(S \Omega-1)+S \beta_{B} S \alpha(S \Omega-1)+C \beta_{B} S \theta C \Omega\right) \\
r_{m}(C \theta C \Omega+S \theta S \Omega-S \theta)+\left(r_{b}-\delta_{B}\right)\left(C \beta_{B} C \alpha C \theta C \Omega-S \beta_{B} S \alpha C \Omega+C \beta_{B} S \theta(S \Omega-1)\right) \\
0
\end{array}\right] \dot{\Omega} } \\
& +\left[\begin{array}{c}
-r_{m}(S \theta C \Omega+C \theta S \Omega) \\
r_{m}(C \theta C \Omega-S \theta S \Omega) \\
r_{m} t \alpha
\end{array}\right] \dot{\theta}+\left[\begin{array}{c}
\left(r_{b}-\delta_{B}\right)\left(C \beta_{B} \omega_{b}-S \beta_{B} \omega_{n}\right) C \Omega \\
\left(r_{b}-\delta_{B}\right)\left(C \beta_{B} \omega_{b}-S \beta_{B} \omega_{n}\right) S \Omega \\
0
\end{array}\right] .
\end{aligned}
$$


Similarly, the velocity of contact point $A_{b}$ on the ball at the contact region of the ball and nut in $X^{\prime}$ system is

$$
\begin{aligned}
V_{A_{b}}= & T_{1} \dot{X}_{A}=\dot{T}_{1} X_{A}+T_{1} \dot{X}_{A} \\
= & \dot{\Omega}\left[\begin{array}{c}
-r_{m}(C \theta S \Omega+S \theta C \Omega)+\left(r_{b}-\delta_{A}\right)\left(C \beta_{A} C \alpha C \theta S \Omega-S \beta_{A} S \alpha S \Omega+C \beta_{A} S \theta C \Omega\right) \\
r_{m}(C \theta C \Omega+S \theta S \Omega)-\left(r_{b}-\delta_{A}\right)\left(C \beta_{A} C \alpha C \theta C \Omega-S \beta_{A} S \alpha C \Omega+C \beta_{A} S \theta S \Omega\right) \\
0
\end{array}\right]+\left[\begin{array}{c}
-r_{m}(S \theta C \Omega+C \theta S \Omega) \\
r_{m}(C \theta C \Omega-S \theta S \Omega) \\
r_{m} t \alpha
\end{array}\right] \dot{\theta} \\
- & {\left[\begin{array}{c}
\left(r_{b}-\delta_{A}\right)\left(C \beta_{A} \omega_{b} C \Omega-S \beta_{A} \omega_{n} C \Omega-S \beta_{A} \omega_{t} S \Omega\right) \\
\left(r_{b}-\delta_{A}\right)\left(C \beta_{A} \omega_{b} S \Omega-S \beta_{A} \omega_{n} S \Omega+S \beta_{A} \omega_{t} C \Omega\right) \\
-\left(r_{b}-\delta_{A}\right) C \beta_{A} \omega_{t}
\end{array}\right] . }
\end{aligned}
$$

In the feed unit of the machine tool, the nut is fixed to the table and can only move along the guide. So, the nut is restricted by the screw but can move in line. In $X^{\prime}$ system, the velocity of the contact point $A_{n}$ on the nut is

$$
\begin{aligned}
V_{A_{n}}= & {\left[\begin{array}{c}
0 \\
0 \\
r_{m} t \alpha-\left(r_{b}-\delta_{A}\right) C \beta_{A} S \alpha S \theta
\end{array}\right] \dot{\theta} } \\
& +\left[\begin{array}{c}
0 \\
0 \\
-\left(C \beta_{A} S \alpha C \theta-S \beta_{A} C \alpha\right)
\end{array}\right] \dot{\delta_{A}} .
\end{aligned}
$$

$$
\begin{aligned}
\Delta V_{A_{b n}}= & \dot{\Omega}\left[\begin{array}{c}
-r_{m}(C \theta S \Omega+S \theta C \Omega)+\left(r_{b}-\delta_{A}\right)\left(C \beta_{A} C \alpha C \theta S \Omega-S \beta_{A} S \alpha S \Omega+C \beta_{A} S \theta C \Omega\right) \\
r_{m}(C \theta C \Omega+S \theta S \Omega)-\left(r_{b}-\delta_{A}\right)\left(C \beta_{A} C \alpha C \theta C \Omega-S \beta_{A} S \alpha C \Omega+C \beta_{A} S \theta S \Omega\right) \\
0
\end{array}\right]+\left[\begin{array}{c}
-r_{m}(S \theta C \Omega+C \theta S \Omega) \\
r_{m}(C \theta C \Omega-S \theta S \Omega) \\
\left(r_{b}-\delta_{A}\right) C \beta_{A} S \alpha S \theta
\end{array}\right] \dot{\theta} \\
& -\left[\begin{array}{c}
\left(r_{b}-\delta_{A}\right)\left(C \beta_{A} \omega_{b} C \Omega-S \beta_{A} \omega_{n} C \Omega-S \beta_{A} \omega_{t} S \Omega\right) \\
\left(r_{b}-\delta_{A}\right)\left(C \beta_{A} \omega_{b} S \Omega-S \beta_{A} \omega_{n} S \Omega+S \beta_{A} \omega_{t} C \Omega\right) \\
-\left(r_{b}-\delta_{A}\right) C \beta_{A} \omega_{t}
\end{array}\right]
\end{aligned}
$$

Because $\delta_{A}$ is a small value, the derivation of $\delta_{A}$, with $\dot{\delta}_{A} \approx 0$, using an approximated expression of (15) is

$$
V_{A_{n}}=\left[\begin{array}{c}
0 \\
0 \\
r_{m} t \alpha-\left(r_{b}-\delta_{A}\right) C \beta_{A} S \alpha S \theta
\end{array}\right] \dot{\theta} .
$$

There is a difference between the nut and ball at the contact point $A$ :
According to the velocity analysis of the ball screw parts, there inevitably exists a relative slide in the ball, screw, and nut. The slide is one of the major factors that cause the wear of the ball screw.

Based on the theory in [6], the velocity of the nut in $z^{\prime}$ direction is

$$
V_{n}=\frac{\dot{\Omega} L}{2 \pi} .
$$

In calculation, the velocity of the nut in $z^{\prime}$ direction is

$$
\left(\frac{L}{2 \pi}-\left(r_{b}-\delta_{A}\right) C \beta_{A} S \alpha S \theta\right) \dot{\theta} .
$$

The velocity of the nut is disturbed by the contact angle, $\beta_{A}$, contact deformation, $\delta_{A}$, relative position, $\theta$, and the velocity, $\dot{\theta}$, of the ball around the screw axis. The variation of the nut velocity will affect the dynamic precision of the table.

\section{Force Analysis of the Hertzian Contact}

The contact problem of the ball screw is the typical rolling contact in $n b$ plane. In the process of the ball moving along the groove, the ball bears four forces: the contact force at points $A, B, q_{A}, q_{B}$ and the friction force at points $A, B, f_{A}, f_{B}$, as shown in Figure 3. Assuming that the friction angles between the friction force and $n b$ plane are $\varphi_{A}, \varphi_{B}$, the forces in $t, n$, and $b$ directions, respectively, are

$$
\begin{aligned}
F_{t}= & f_{A} q_{A} \sin \varphi_{A}-f_{B} q_{B} \sin \varphi_{B}, \\
F_{n}= & -q_{A} \cos \beta_{A}+f_{A} q_{A} \cos \varphi_{A} \sin \beta_{A}+q_{B} \cos \beta_{B} \\
& -f_{B} q_{B} \cos \varphi_{B} \sin \beta_{A},
\end{aligned}
$$




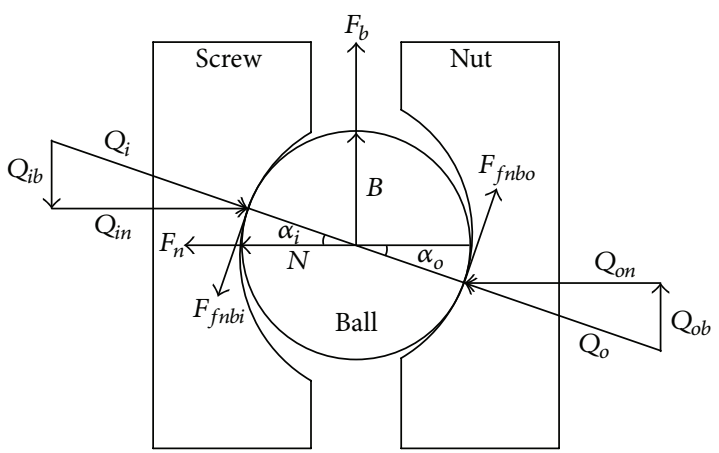

FIGURE 3: The force analysis of the ball in the normal plane of the Frenet coordinate system.

$$
\begin{aligned}
F_{b}= & -q_{A} \sin \beta_{A}-f_{A} q_{A} \cos \varphi_{A} \cos \beta_{A}+q_{B} \sin \beta_{B} \\
& +f_{B} q_{B} \cos \varphi_{B} \cos \beta_{A} .
\end{aligned}
$$

From the force analysis presented in [6],

$$
\begin{aligned}
& F_{t}=m \frac{r_{m}}{\cos \alpha}\left(\ddot{\theta}+\cos ^{2} \alpha\right), \\
& F_{n}=m r_{m}(\dot{\theta}+\dot{\Omega})^{2}, \\
& F_{b}=m r_{m} \sin \alpha \ddot{\Omega} .
\end{aligned}
$$

Assuming an axis load, $F_{a}$, is implemented on the nut, $F_{a}$ will be distributed onto every ball without considering the machining error of the groove. The force on the contact point of one ball and the nut is

$$
\begin{aligned}
& \frac{F_{a}}{z} \\
& \quad \\
& \quad=q_{A} \sin \beta_{A} \cos \alpha-f_{A} q_{A} \sin \varphi_{A} \sin \alpha \\
& \quad+f_{A} q_{A} \cos \varphi_{A} \cos \beta_{A} \cos \alpha, \\
& \frac{q_{A}+F_{n} \cos \beta_{A}+F_{b} \sin \beta_{A}}{\left(\cos \beta_{B} \cos \beta_{A}+\sin \beta_{B} \sin \beta_{A}\right)}=q_{B},
\end{aligned}
$$

where $z$ is the number of balls in the nut.

The contact situation of the ball and screw (nut), which is similar to the bearing, can be represented by two semiellipsoids whose corresponding planes of principal curvature through the point of contact coincide and whose radii of curvature in those planes have the same values as those of the unloaded original solids at the contact point (as shown in Figure 4). The relative curvatures in $x z$ plane and $y z$ plane are

$$
\begin{aligned}
& \frac{1}{R_{x}}=\frac{1}{r_{1 x}}+\frac{1}{r_{2 x}}, \\
& \frac{1}{R_{y}}=\frac{1}{r_{1 y}}+\frac{1}{r_{2 y}},
\end{aligned}
$$
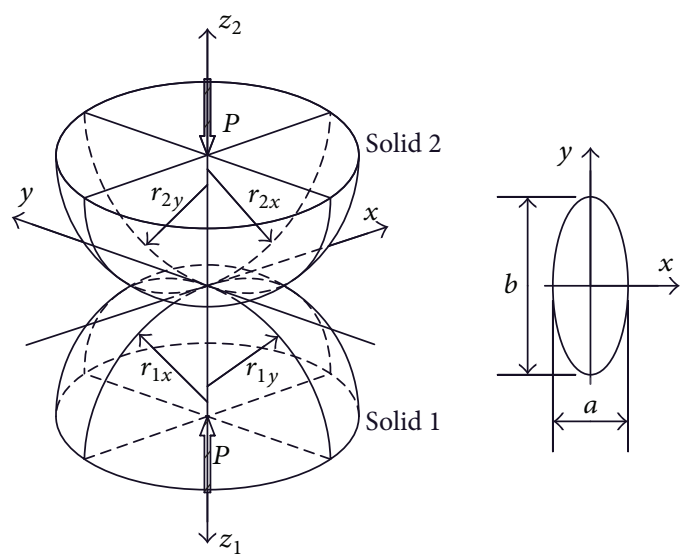

FIGURE 4: The shape of the contact area between the ball and the nut (screw) in Hertzian contact theory.

where $r_{i x}, r_{i y}$ are principal radii of the curvature of body 1 in $x z$ plane and $y z$ plane, respectively. The effective radius ratio and the ellipticity are

$$
\begin{aligned}
& \gamma=\frac{R_{y}}{R_{x}}, \\
& \kappa=\frac{b}{a},
\end{aligned}
$$

where $a, b$ are the diameters of the contact ellipses parallel to $x z$ plane and $y z$ plane, respectively.

In the contact form of the ball and nut (screw), the curvature sum is

$$
\frac{1}{R}=\frac{1}{R_{x}}-\frac{1}{R_{y}} .
$$

According to the Hertzian contact theory [13], the relationship between the deformation and the normal force is

$$
\delta=\frac{\left\{(2 \varsigma / \pi)\left(\pi / 2 \kappa^{2} \xi\right)^{1 / 3}\right\}\left(3 P R / E^{\prime}\right)^{2 / 3}}{2 R}
$$

where $E^{\prime}=2 /\left(\left(1-v_{1}\right) / E_{1}+\left(1-v_{2}\right) / E_{2}\right), \varsigma=\int_{0}^{\pi / 2}\{1-(1-$ $\left.\left.\kappa^{-2}\right) \sin ^{2} \theta\right\}^{-1 / 2} d \theta, \xi=\int_{0}^{\pi / 2}\left\{1-\left(1-\kappa^{-2}\right) \sin ^{2} \theta\right\}^{1 / 2} d \theta, E_{i}$ is the elastic modulus of the contact body material, and $\nu_{i}$ is the Poisson ratio.

The precision of the parameter approximate formula for $\varsigma, \xi$ is simulated in [12]. The minimum error formulas are chosen as follows:

$$
\begin{aligned}
& \kappa=1.03 \gamma^{0.636}, \\
& \varsigma=1.5277+1.6023 \ln \gamma, \\
& \xi=1.0003+0.5968 \gamma^{-1} .
\end{aligned}
$$




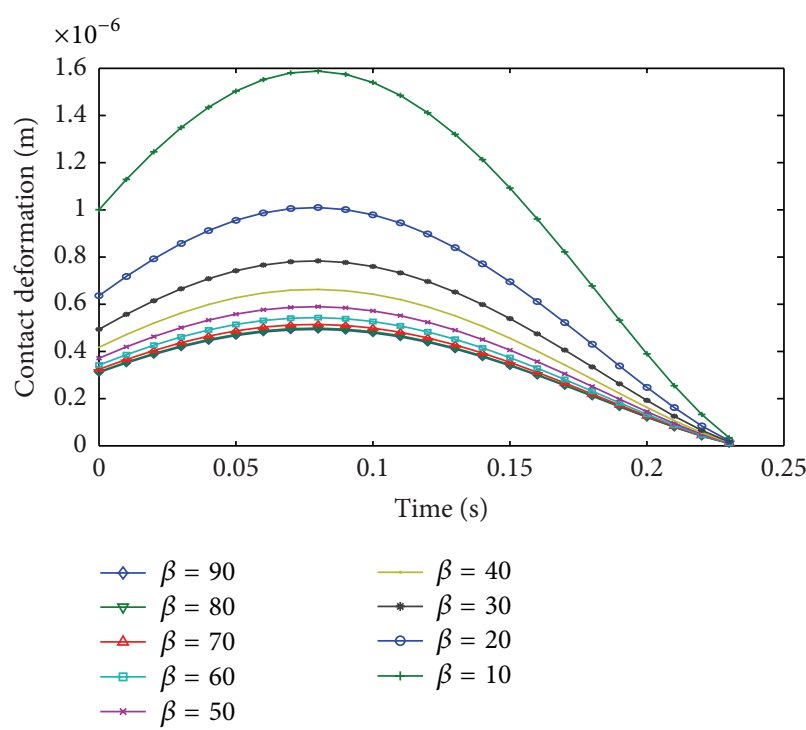

(a)

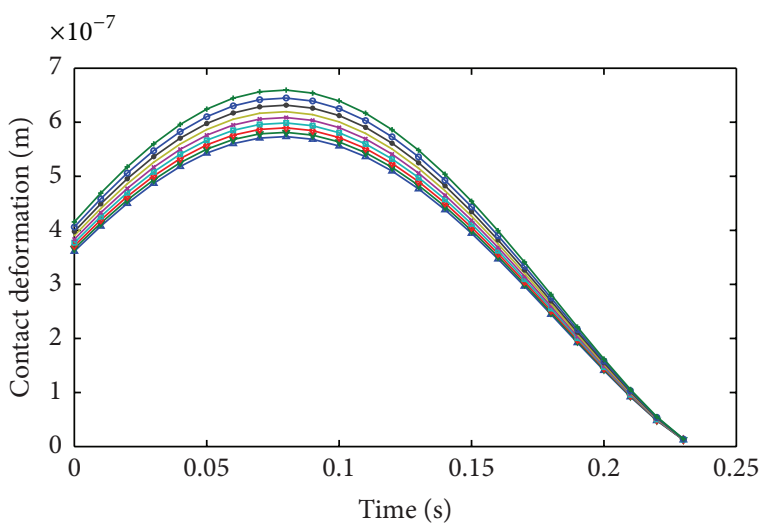

$\rightarrow E_{1}=2.1 * 10^{11} \mathrm{~N} / \mathrm{m}^{2} E_{2}=2.4 * 10^{11} \mathrm{~N} / \mathrm{m}^{2}$
$\rightarrow-E_{1}=2.1 * 10^{11} \mathrm{~N} / \mathrm{m}^{2} E_{2}=2.3 * 10^{11} \mathrm{~N} / \mathrm{m}^{2}$
$\rightarrow-E_{1}=2.1 * 10^{11} \mathrm{~N} / \mathrm{m}^{2} E_{2}=2.2 * 10^{11} \mathrm{~N} / \mathrm{m}^{2}$
$\square-E_{1}=2.1 * 10^{11} \mathrm{~N} / \mathrm{m}^{2} E_{2}=2.2 * 10^{11} \mathrm{~N} / \mathrm{m}^{2}$
$\rightarrow-E_{1}=2.1 * 10^{11} \mathrm{~N} / \mathrm{m}^{2} E_{2}=2.1 * 10^{11} \mathrm{~N} / \mathrm{m}^{2}$
$\rightarrow-E_{1}=2.1 * 10^{11} \mathrm{~N} / \mathrm{m}^{2} E_{2}=2.0 * 10^{11} \mathrm{~N} / \mathrm{m}^{2}$
$\rightarrow-E_{1}=2.1 * 10^{11} \mathrm{~N} / \mathrm{m}^{2} E_{2}=1.9 * 10^{11} \mathrm{~N} / \mathrm{m}^{2}$
$\rightarrow-E_{1}=2.1 * 10^{11} \mathrm{~N} / \mathrm{m}^{2} E_{2}=1.8 * 10^{11} \mathrm{~N} / \mathrm{m}^{2}$
$\rightarrow-E_{1}=2.1 * 10^{11} \mathrm{~N} / \mathrm{m}^{2} E_{2}=1.7 * 10^{11} \mathrm{~N} / \mathrm{m}^{2}$

(b)

FIgURE 5: (a) The influence of the contact angle on the deformation. (b) The influence of the elastic modal on the deformation.

By taking (22) and (28)-(30) into (27), the relationship formula changes to

$$
\begin{aligned}
& \delta_{A} \\
& =\frac{\left\{\left(\varsigma / \pi^{2 / 3}\right)\left(1 / 2 \kappa^{2} \xi\right)^{1 / 3}\right\}\left(3 F_{a} / E^{\prime} z\left(\sin \beta_{A} \cos \alpha-f_{A} \sin \alpha\right)\right)^{2 / 3}}{R^{1 / 3}} .
\end{aligned}
$$

Hence, the positioning error of the table caused by the contact deformation is

$$
\delta_{z^{\prime}}=\left(\delta_{B}+\delta_{A}\right) S \beta_{A} C \alpha
$$

\section{Simulation of the Contact Deformation}

From formula (30), we know that the contact deformation is related to the helical angle $(\alpha)$, the contact angle $\left(\beta_{A}\right)$, the number of balls $(z)$, the curvature sum $(R)$, the equivalent elastic modulus $(E)$, the effective radius ratio $(\gamma)$, the friction force, and the load. The relationship between the helical angle $(\alpha)$, the contact angle $\left(\beta_{A}\right)$, the equivalent elastic modulus $(E)$, the load, and the contact deformation is studied using a simulation.

The simulation geometry and material parameters of the ball screw are listed in Table 1. The equation, $F=A(1+$ $\sin \omega_{f} t$ ), is used as the disturbance load, with $\omega=20 \mathrm{~Hz}$. Firstly, the contact angle is increased from $10^{\circ}$ to $90^{\circ}$ and $A=$ $100 \mathrm{~N}$. The variation of the contact deformation caused by the change in the contact angle is shown in Figure 5(a). Next, Young's modulus of the nut is changed from $1.6 \times 10^{11} \mathrm{~N} / \mathrm{m}^{2}$ to $2.4 \times 10^{11} \mathrm{~N} / \mathrm{m}^{2}$ by the step size of $0.1 \times 10^{11} \mathrm{~N} / \mathrm{m}^{2}$.

\begin{tabular}{|c|c|c|c|}
\hline \multirow{2}{*}{ Parameter } & \multicolumn{2}{|c|}{ Value } & \multirow{2}{*}{ Unit } \\
\hline & Nut & Screw & \\
\hline Helical angle & \multicolumn{2}{|c|}{2.967} & Degree \\
\hline Pitch & \multicolumn{2}{|c|}{6} & $\mathrm{~mm}$ \\
\hline Ball radius & \multicolumn{2}{|c|}{1.5875} & $\mathrm{~mm}$ \\
\hline Number of balls & \multicolumn{2}{|c|}{71} & - \\
\hline Young's modulus & $2.10 \times 10^{11}$ & $2.09 \times 10^{11}$ & $\mathrm{~N} / \mathrm{m}^{2}$ \\
\hline Poisson ratio & 0.278 & 0.295 & - \\
\hline Relative curvature $R_{x}$ & \multicolumn{2}{|c|}{1.4588} & $\mathrm{~mm}$ \\
\hline Relative curvature $R_{y}$ & \multicolumn{2}{|c|}{1.4588} & $\mathrm{~mm}$ \\
\hline
\end{tabular}
The simulation result is shown in Figure 5(b). Finally, the
TABLE 1: Geometry and material parameters of ball screw.

relationship between the contact deformation and the load, which could be called contact stiffness, is simulated as shown in Figure 6. The load varies from $0 \mathrm{~N}$ to $2000 \mathrm{~N}$, the contact angle is $48.5^{\circ}$, and Young's modulus values are listed in Table 1.

As shown in Figure 5(a), the relationship between the contact angle and the contact deformation is nonlinear. When the angle is greater than $50^{\circ}$, the changes in the angle have no obvious impact on the deformation. However, the deformation will increase quickly if the angle is smaller than $50^{\circ}$. The relationship between Young's modulus and the contact deformation is nonlinear as well (Figure 5(b)). Increasing the value of the equivalent elastic modulus which is decided by the smaller modulus could decrease the deformation. The socalled contact stiffness of the ball screw is nonlinear, as shown in Figure 6 . The deformation will increase to $\mu \mathrm{m}$ magnitude, which will increase the machining error in the high-speed high-precision machine tool. 


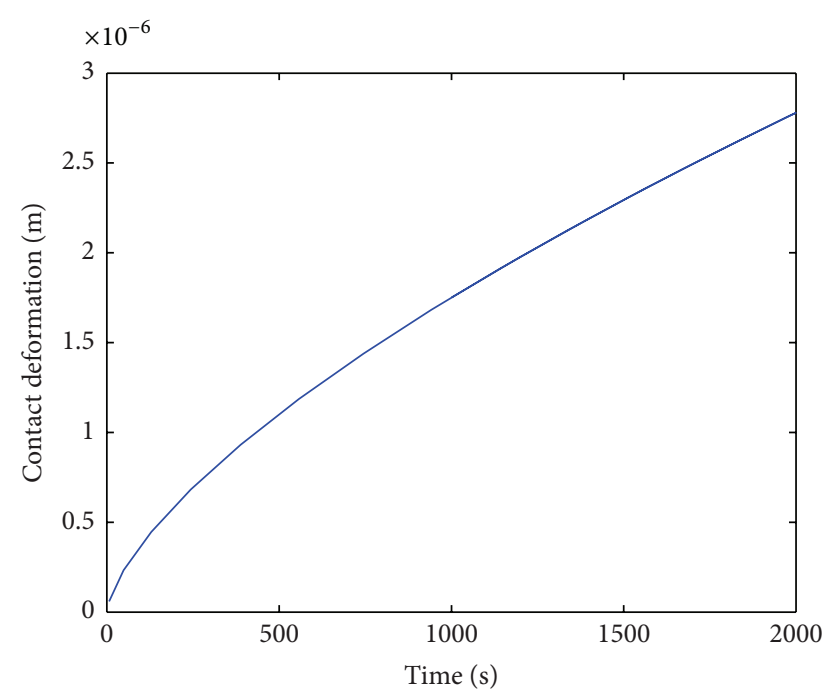

FIGURE 6: The relationship between the force and the deformation.

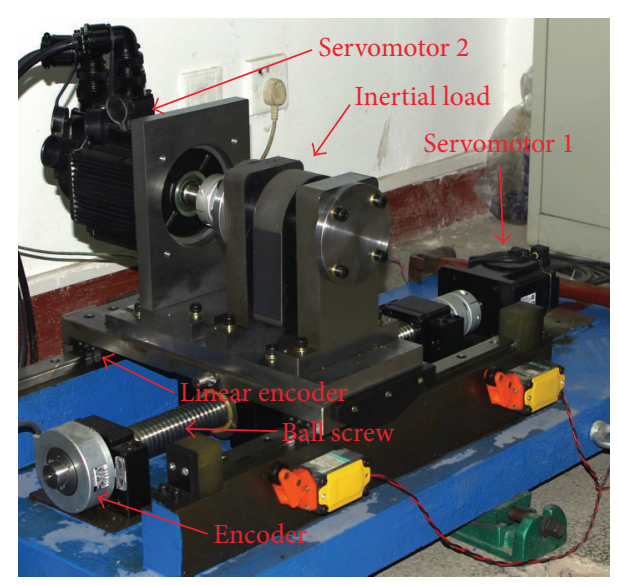

FIgURE 7: The ball screw drive set-up.

\section{Experiment of the Contact Deformation with Load Disturbance}

To confirm the influence of the contact deformation on the transmission precision of the ball screw, experiments are implemented on a ball screw test bed (shown in Figure 7). On the test bed, a double nut, no-backlash ball screw is used as the experimental object, which has a type number of BNFN-3606-2.5. An open control card is used to drive the servomotor. An encoder is installed at the end of the screw, and a linear encoder is used in the bed to provide feedback of the position of the nut (table). An inertial loading unit is used to simulate the load disturbance. The amplitude and frequency are controlled by the speed of the motor installed on the unit. The relationship between inertia torque and speed is

$$
F_{n}=0.143 \times 10^{-2} \times n_{r}^{2}
$$

where $n_{r}$ is the speed of the motor.
The encoder mounted at the end of the screw, which has a precision of $0.58 \mu \mathrm{m}$ in the axial direction, and the linear encoder, whose precision is $0.1 \mu \mathrm{m}$, are used to test the deformation of the contact region. The data acquisition instrument used here was developed by the research group based on the IK220 counter card. The deformation of the screw rotation, which is restricted under the static condition, is tested first. The speed of the inertial loading unit increases from $30 \mathrm{r} / \mathrm{min}$ to $330 \mathrm{r} / \mathrm{min}$ by a step of $10 \mathrm{r} / \mathrm{min}$. In other words, the peak of the simulated load disturbance increases from $1.287 \mathrm{~N}$ to $155.72 \mathrm{~N}$. The signals of the end encoder and the linear encoder are sampled synchronously with a $1000 \mathrm{~Hz}$ sampling frequency, and part of the sampling signal, when tested at $200 \mathrm{r} / \mathrm{min}$, is shown in Figure $8(\mathrm{a})$. The amplitude of the contact deformation is on $\mu \mathrm{m}$ scale under $200 \mathrm{r} / \mathrm{min}$ conditions, and there exists a phase delay between the encoder and the linear encoder. The signal frequency of the tested signals is the same as the rotation frequency of the inertia unit. Both of the amplitudes of the contact deformation tested in each speed are used to determine the relationship between the load disturbance and the contact deformation, as shown in Figure 8(b). The contact stiffness curves that were tested at the table and at the screw are generally consistent with each other when the load is greater than $65 \mathrm{~N}$. In the range of $0 \mathrm{~N}$ to $65 \mathrm{~N}$, the curves have similar trends; however, the amplitude deviation is comparatively large. The amplitude of the tested stiffness curve is a little bigger than the simulation result.

Another test is implemented to test the dynamic precision of the ball screw with the load disturbance. The straight reciprocating motion of the ball screw with a speed of $10 \mathrm{~mm} / \mathrm{s}$ is tested. In the process, the speed inertia is set to $0 \mathrm{r} / \mathrm{min}, 200 \mathrm{r} / \mathrm{min}$, and $300 \mathrm{r} / \mathrm{min}$, respectively, and the data acquisition equipment is the same as that used for the static test. The test results are shown in Figure 9. The fluctuation frequency, which is the rotational frequency of the inertia unit of the table position, is $3.33 \mathrm{~Hz}$ under the $200 \mathrm{r} / \mathrm{min}$ condition and $5.003 \mathrm{~Hz}$ in $300 \mathrm{r} / \mathrm{min}$. The fluctuation frequencies clearly show the influence of the load disturbance on the position precision. However, the amplitude is approximately ten times that tested in the static condition. This result is due to the other sources of error, such as the pitch error, and the axial deformation of the screw may be included in the data.

\section{Conclusions}

High-speed and high-precision NC machine tools are critical for modern industry. To improve the dynamic machining precision of NC machine tool, the transmission process of the ball screw is studied in this paper. Rigid-body kinematics are used to analyze the static part of the ball screw by considering contact deformation. Additionally, the Hertzian contact theory is used to analyze the influence factor on contact deformation. The relationships among the load disturbance, the deformation, and the influence factor are studied by simulation and experiment. The results show that the contact deformation indeed partially impacts the motion precision. The relationship between the load disturbance and the deformation is nonlinear, which leads to so-called 


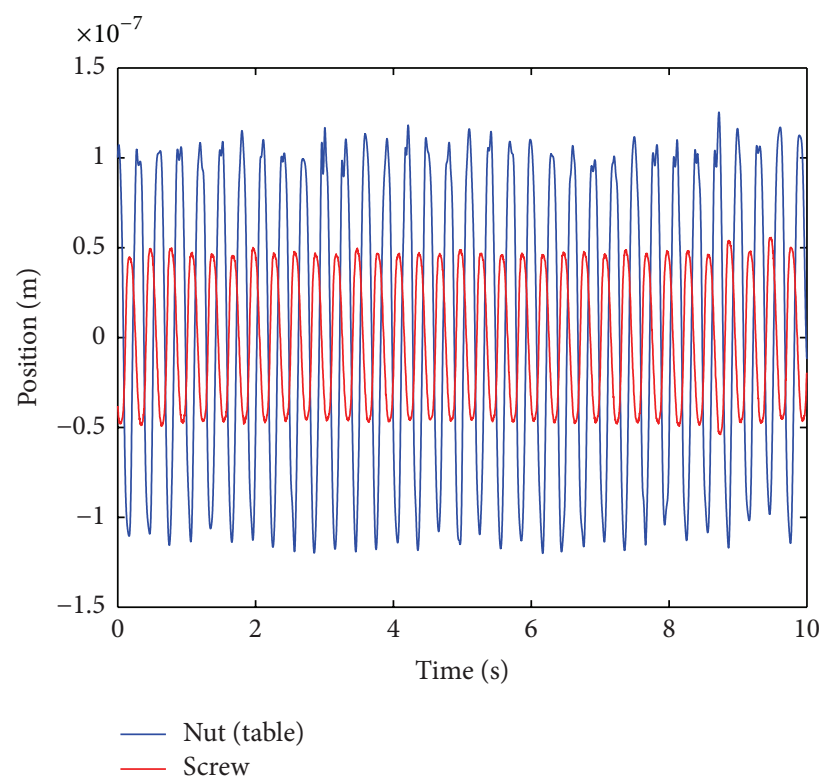

(a)

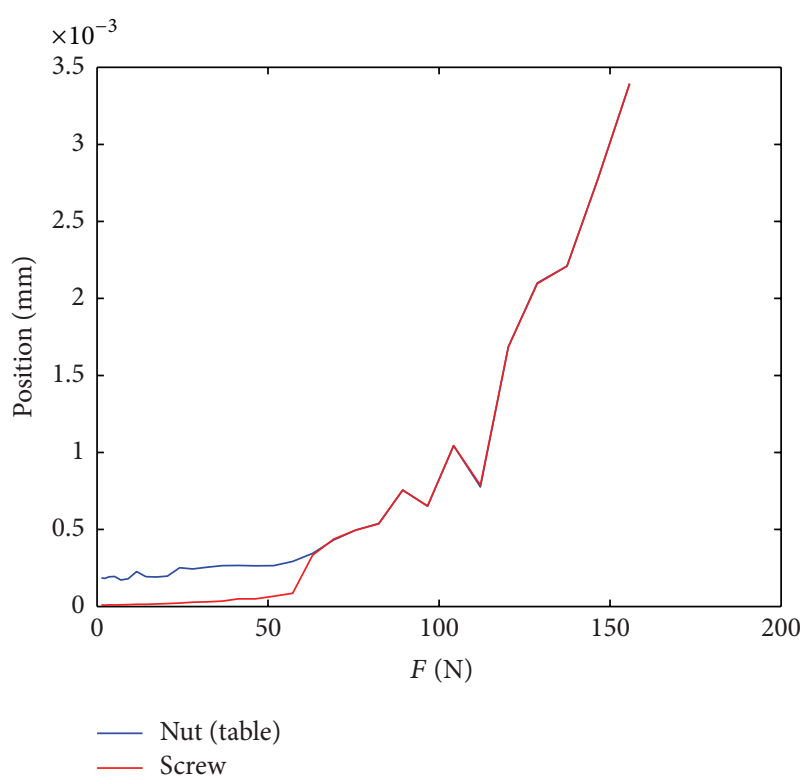

(b)

Figure 8: (a) The position signals of the linear encoder and the encoder in a static test $(r=200 \mathrm{r} / \mathrm{min})$. (b) The test result of the relationship between force and deformation.

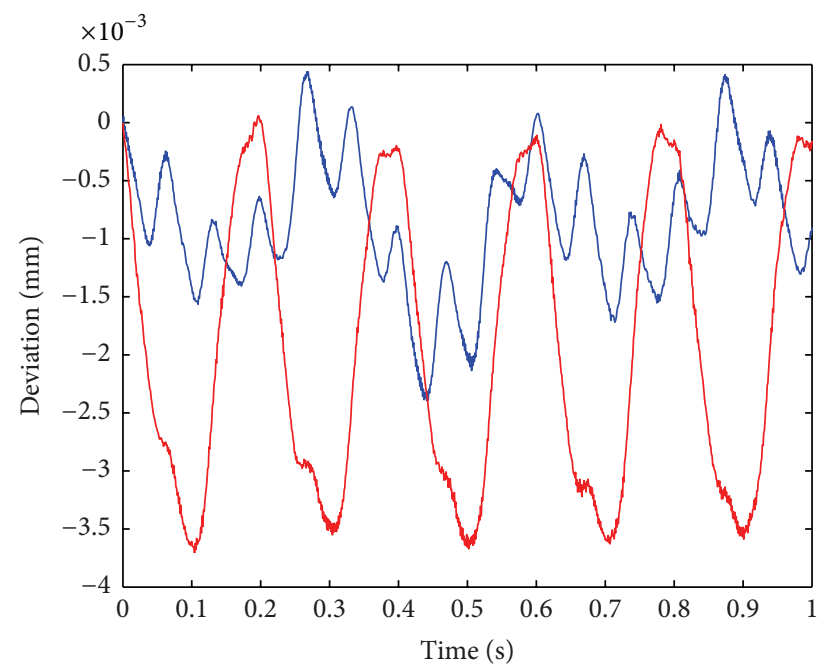

FIGURE 9: The tested signals of the linear encoder when moving under the applied force.

contact stiffness. The relationship is influenced by the contact angle and the material characteristics, whereas the helical angle has little influence. When the amplitude of the load disturbance is close to $1000 \mathrm{~N}$, the contact deformation will not be ignored in the feed system of a high-speed and highprecision machine tool.

\section{Notations}

$a$ : The diameters of the contact ellipse parallel to $x z$ plane

$b$ : The diameters of the contact ellipse parallel to $y z$ plane
$A_{b}$ : Contact point on ball at the contact region of ball and nut

$A_{n}$ : Contact point on nut at the contact region of ball and nut

$B_{s}$ : Contact point on screw at the contact region of ball and screw

$B_{b}$ : Contact point on ball at the contact region of ball and screw

$E_{i}$ : The elastic modulus of the contact body material

$f_{A}:$ Friction force at point $A$

$f_{B}$ : Friction force at point $B$

$F_{t}$ : External force component in $t$ direction

$F_{n}$ : External force component in $n$ direction

$F_{b}$ : External force component in $b$ direction

$F_{a}$ : Load of the nut

$L$ : The pitch of screw

$n_{r}$ : The speed of motor

${ }^{r} P$ : The coordinate of the ball center in $X$ system

${ }^{w} P$ : The coordinate of the ball center in $X^{\prime}$ system

$q_{A}:$ Normal contact force at point $A$

$q_{B}$ : Normal contact force at point $B$

$r_{m}$ : The nominal radius of screw

$R_{x}$ : Relative curvature in $x z$ plane

$r_{1 x}$ : Principal radii of curvature of body 1 in $x z$ plane

$r_{2 x}$ : Principal radii of curvature of body 2 in $x z$ plane

$R_{y}:$ Relative curvature in $y z$ plane

$r_{1 y}$ : Principal radii of curvature of body 12 in $y z$ plane

$r_{2 y}$ : Principal radii of curvature of body 2 in $y z$ plane 


\begin{tabular}{|c|c|}
\hline$T_{1}:$ & Transmission function from $X$ to $X^{\prime}$ \\
\hline$V_{B_{b}}:$ & Velocity of the contact point $B_{b}$ on ball \\
\hline$V_{B_{s}}:$ & Velocity of the contact point on screw $B_{s}$ \\
\hline$\Delta V_{B_{b s}}^{s}:$ & $\begin{array}{l}\text { Deviation between the ball velocity and } \\
\text { screw at the contact point } B\end{array}$ \\
\hline$V_{A_{h}}:$ & Velocity of the contact point $A_{b}$ on ball \\
\hline$V_{A_{n}}:$ & Velocity of the contact point $A_{n}$ on nut \\
\hline$\Delta V_{A_{b n}}:$ & $\begin{array}{l}\text { Deviation between the ball velocity and } \\
\text { screw at the contact point } A\end{array}$ \\
\hline$V_{n}:$ & The velocity of nut in theory \\
\hline$X^{\prime}\left(x^{\prime}, y^{\prime}, z^{\prime}\right)$ & The first coordinate system \\
\hline$X(x, y, z):$ & The second coordinate system \\
\hline$Y(t, n, b):$ & The Frenet coordinate system \\
\hline$z:$ & The number of balls in nut \\
\hline$\alpha:$ & Helical angle of groove \\
\hline$\beta_{i}(i=A, B):$ & The contact angle at point $A, B$ \\
\hline$\delta:$ & The contact deformation \\
\hline$\delta_{z^{\prime}}:$ & $\begin{array}{l}\text { The component of the contact deformation } \\
\text { in } z^{\prime} \text { direction }\end{array}$ \\
\hline$\varphi_{A}:$ & $\begin{array}{l}\text { The friction angle between friction force and } \\
n b \text { plane at point } A\end{array}$ \\
\hline$\varphi_{B}:$ & $\begin{array}{l}\text { The friction angle between friction force and } \\
n b \text { plane at point } B\end{array}$ \\
\hline$\kappa:$ & The ellipticity of the contact area \\
\hline$\gamma:$ & The effective radius ratio of the contact area \\
\hline$v_{i}:$ & Poisson ratio of the contact body material \\
\hline$\dot{\theta}:$ & The velocity of balls around $x$ axis \\
\hline$\ddot{\theta}:$ & The acceleration of balls around $x$ axis \\
\hline$\varsigma:$ & $\begin{array}{l}\text { The complete elliptic integrals of the first } \\
\text { kind }\end{array}$ \\
\hline$\omega_{i}($ & $\begin{array}{l}\text { The angular velocity of balls around the } \\
t, n, b \text { axes }\end{array}$ \\
\hline$\dot{\Omega}:$ & The angular velocity of screw around $z^{\prime}$ axis \\
\hline$\ddot{\Omega}:$ & $\begin{array}{l}\text { The angular acceleration of screw around } z^{\prime} \\
\text { axis }\end{array}$ \\
\hline$\xi$ & The complete elliptic integrals of the second \\
\hline
\end{tabular}

\section{Competing Interests}

The authors declare that they have no competing interests.

\section{Acknowledgments}

This work was supported by the National Important Special Project under Grants [2015ZX04005001], Errors Measurement, Analysis and Compensation of CNC Machine Tools.

\section{References}

[1] G. Pritschow, "A comparison of linear and conventional electromechanical dives," CIRP Annals-Manufacturing Technology, vol. 47, no. 2, pp. 541-548, 1998.

[2] D. A. Vicente, R. L. Hecker, and G. M. Flores, "Ball screw drive systems: evaluation of axial and torsional deformations," Mecánica Computacional, vol. 28, no. 38, pp. 3265-3277, 2009.

[3] K. K. Varanasi and S. A. Nayfeh, "The dynamics of leadscrew drives: low-order modeling and experiments," Journal of
Dynamic Systems, Measurement and Control, vol. 126, no. 2, pp. 388-396, 2004.

[4] C. E. Okwudire, "Improved screw-nut interface model for highperformance ball screw drives," Journal of Mechanical Design, vol. 133, no. 4, Article ID 041009, 10 pages, 2011.

[5] C. E. Okwudire and Y. Altintas, "Hybrid modeling of ball screw drives with coupled axial, torsional, and lateral dynamics," Journal of Mechanical Design, vol. 131, no. 7, pp. 07100210710029, 2009.

[6] M. C. Lin, B. Ravani, and S. A. Velinsky, "Kinematics of the ball screw mechanism," Journal of Mechanical Design, vol. 116, no. 3 , pp. 849-855, 1994.

[7] M. C. Lin, S. A. Velinsky, and B. Ravani, "Design of the ball screw mechanism for optimal efficiency," Journal of Mechanical Design, vol. 116, no. 3, pp. 856-861, 1994.

[8] C. C. Wei and J. F. Lin, "Kinematic analysis of the ball screw mechanism considering variable contact angles and elastic deformations," Journal of Mechanical Design, vol. 125, no. 4, pp. 717-733, 2003.

[9] G.-H. Feng and Y.-L. Pan, "Investigation of ball screw preload variation based on dynamic modeling of a preload adjustable feed-drive system and spectrum analysis of ball-nuts sensed vibration signals," International Journal of Machine Tools and Manufacture, vol. 52, no. 1, pp. 85-96, 2012.

[10] H.-T. T. Huang and B. Ravani, "Contact stress analysis in ball screw mechanism using the tubular medial axis representation of contacting surfaces," Journal of Mechanical Design, vol. 119, no. 1, pp. 8-14, 1997.

[11] J. F. Cuttino, T. A. Dow, and B. F. Knight, "Analytical and experimental identification of nonlinearities in a single-nut, preloaded ball screw," Journal of Mechanical Design, vol. 119, no. 1, pp. 15-19, 1997.

[12] X. Mei, M. Tsutsumi, T. Tao, and N. Sun, "Study on the load distribution of ball screws with errors," Mechanism and Machine Theory, vol. 38, no. 11, pp. 1257-1269, 2003.

[13] P. H. Markho, "Highly accurate formulas for rapid calculation of the key geometrical parameters of elliptic Hertzian contacts," Journal of Tribology, vol. 109, no. 4, pp. 640-647, 1987. 


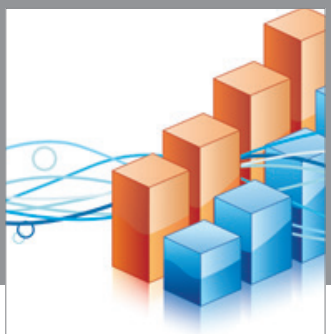

Advances in

Operations Research

vatem alat4



\section{The Scientific} World Journal

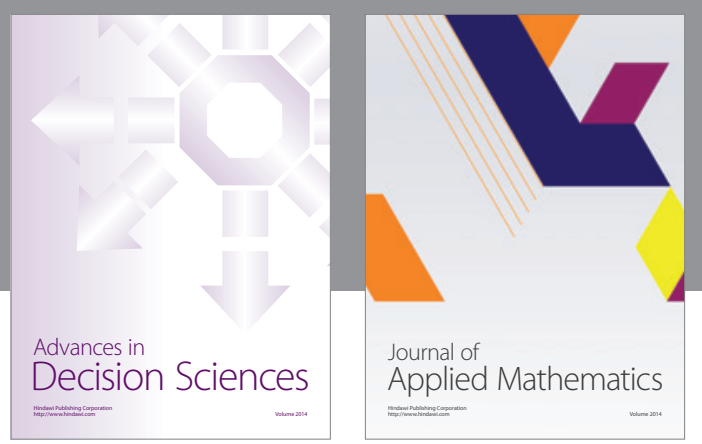

Algebra

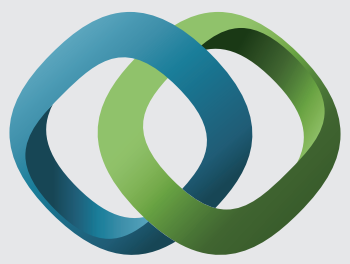

\section{Hindawi}

Submit your manuscripts at

http://www.hindawi.com
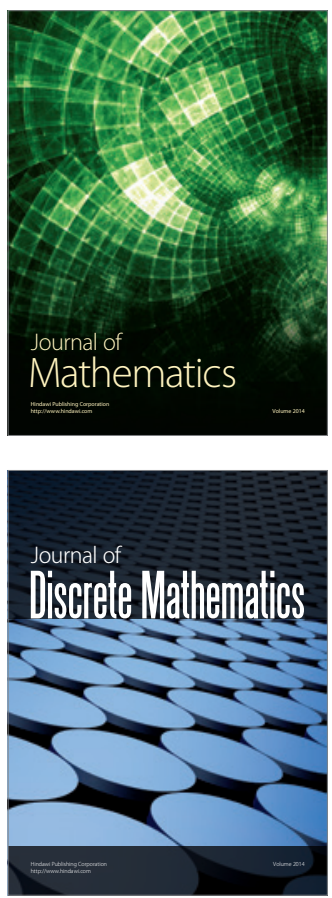

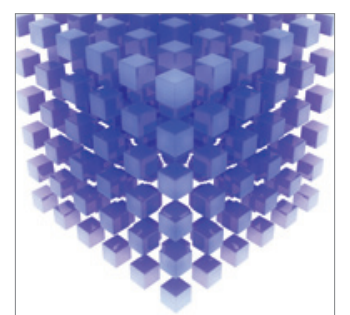

Mathematical Problems in Engineering


Journal of

Function Spaces

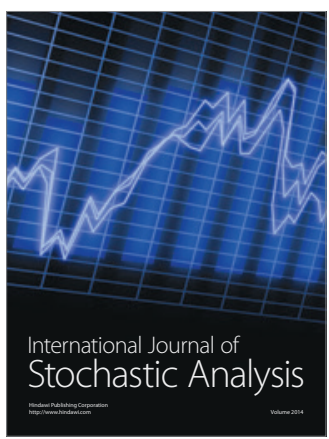

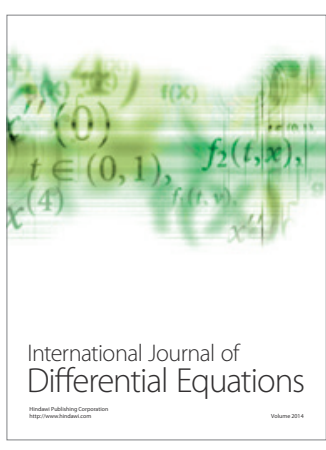
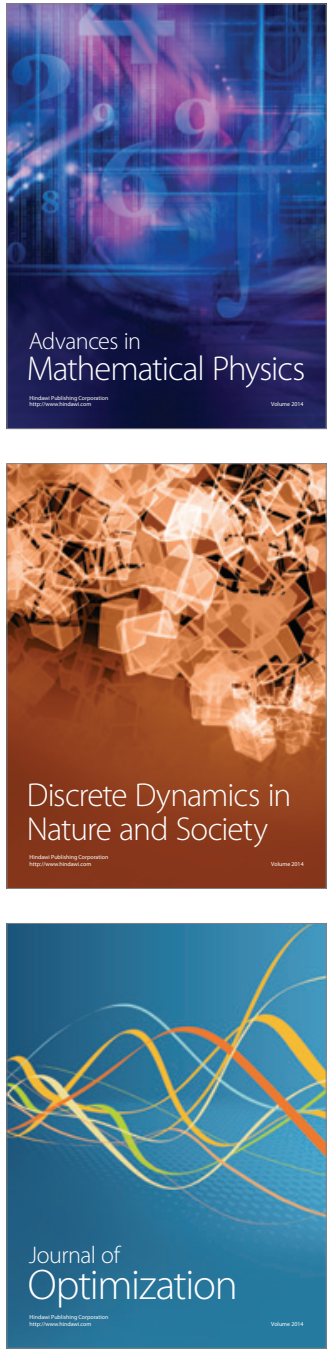